\title{
A new method for accurate platelet thrombi volume measurement using a confocal microscope
}

\author{
Denise De Zanet ${ }^{1,2}$, Monica Battiston ${ }^{2}$, Elisabetta Lombardi ${ }^{2}$, Ruben Specogna ${ }^{1}$, Francesco Trevisan ${ }^{1}$, \\ Luigi De Marcoㄹ, Antonio Affanni ${ }^{1}$, Mario Mazzucato²
}

1 Polytechnic Department of Engineering and Architecture, University of Udine, Italy

2 Department of Translational Research, Stem Cells Unit, National Cancer Institute CRO - IRCCS, Aviano, Italy

\section{ABSTRACT}

The accuracy of quantitative measurements represents an essential pre-requisite to the characterization and definition of the complex dynamic phenomena occurring in the field of cell biology. In research projects that involve the induction of blood coagulation under flow in microfluidic artificial channels, thrombus volume is an important quantity for estimation as a significant index related to the individual thrombotic risk profile. Concerning its importance in the early diagnosis of cardiovascular diseases, the estimated thrombus volume should reflect and represent reality. In 3D confocal microscopy, systematic errors can arise from distortions of the axial distance, whose accurate calibration remains a challenge. As a result, the 3D reconstructions show a noticeable axial elongation, and the volume measurements are thus overestimated. In this paper, a 400-600 \% volume overestimation is demonstrated, and a new easy to use and automatic calibration procedure is outlined for this specific microfluidic and optical context. The adaptive algorithm proposed leads to the automatic compensation of the elongation error and to the accurate thrombus volume measurement. The method has been calibrated using fluorescent beads of known volume, validated with groups of several distinct platelets and finally applied on platelet thrombi.

\section{Section: RESEARCH PAPER}

Keywords: image processing; volume measurement; calibration; blood coagulation; confocal microscopy

Citation: Denise De Zanet, Monica Battiston, Elisabetta Lombardi, Ruben Specogna, Francesco Trevisan, Luigi De Marco, Antonio Affanni, Mario Mazzucato, A new method for accurate platelet thrombi volume measurement using a confocal microscope, Acta IMEKO, vol. 8, no. 1, article 8, March 2019, identifier: IMEKO-ACTA-08 (2019)-01-08

Section Editor: Rosario Morello, University Mediterranea of Reggio Calabria, Italy

Received January 24, 2018; In final form January 19, 2019; Published March 2019

Copyright: (C) 2019 IMEKO. This is an open-access article distributed under the terms of the Creative Commons Attribution 3.0 License, which permits unrestricted use, distribution, and reproduction in any medium, provided the original author and source are credited.

Funding: This work was supported by grant from the Italian Ministry of Health RF-2010-2316198 and RF-2010-317993 (L.D.M., M.M.).

Corresponding author: Antonio Affanni, e-mail: antonio.affanni@uniud.it, Denise De Zanet, e-mail: dezanet@cro.it

\section{INTRODUCTION}

Live-cell microscopy has become essential in the analysis and reconstruction of the intra- and inter-cellular dynamic processes. The numerical approach to these events represents the basis of the emerging studies about medical science. Advances in technologies and computational methods have moved cell biology gradually toward a quantitative view [1]. Numerical computation offers to medicine the possible benefit of quantifying and assessing a broad variety of diseases and medical conditions by means of innovative automated methods of image analysis [2]-[5]. In an attempt to measure cellular functions and morphological features, samples can be studied by using various microscopy techniques [6]. If the specific aim is to measure the volumetric structure of an object, a confocal microscope provides, as output, a sequence of axial fluorescent images that should facilitate three-dimensional volume reconstruction and measurement, rejecting the out-of-focus fluorescent light [7]. However, digital fluorescent images show misrepresented objects because they are modified and transformed by the acquisition system. Therefore, the advanced technology of confocal microscopes hides its limitations, which are often difficult to clearly identify and adjust as well as move biologists far from accurate and realistic measurements [7], [8].

Blood coagulation is a complex mechanism whose alteration can produce diseases such as thrombosis, embolisms, and haemorrhages. In particular, thrombosis is one of the major causes of death and implicates a series of morphological and functional changes in different temporal and spatial dimensional scales. Platelets have a central haemostatic role due to their ability to rapidly adhere to a damaged substrate and to activate and aggregate forming thrombi. Improvements in 
microscope technologies have enabled the investigation of platelet functionality and thrombus formation starting from fluorescent confocal images. However, errors are introduced by the optical system due to a lack of convergence between marginal and paraxial rays and by the mismatch of refractive indexes between the immersion medium and the biological object at the interface. Consequently, spherical aberration phenomena lead to a misrepresentation of the objects, and their effects can be shown as an axial elongation in the threedimensional reconstructions [7], [9]-[12].

In specific scientific literature (e.g. in [13]-[19]) starting from sequences of confocal images acquired along the axial \%dimension, thrombus volume has often been measured without due attention given to the presence of an intrinsic inaccuracy and distortions that have not been compensated for. This inaccuracy has never represented a problem because thrombus volume quantification has always aimed for a comparison between an untreated situation (control) and variations from it due to the effects of biological or pharmaceutical treatments. However, at the same time, medical research has gradually moved its attention to new non-invasive and real-time devices with the aim of extracting and monitoring the individual thrombotic risk profile and the whole dynamic of thrombus formation events [20]-[31]. Some of these new technologies use confocal microscope results as a reference to calibrate their system numerical outputs; however, in this specific context, standardisation and reproducibility still remain open and unresolved issues [32], [33].

Herein, a 400-600\% volume overestimation using available post-processing software is demonstrated. Hence, in order to improve measurement accuracy, a new easy to use and fast calibration method is proposed. An adaptive automatic algorithm is introduced. It allows standardised and accurate volume quantification and a realistic morphological 3D reconstruction starting from the fluorescence intensity signals of the images. The algorithm is calibrated using fluorescent beads of known volume, and it is then applied to platelet aggregates. Accuracy is evaluated, and the related improvement is quantified.

The paper is organised as follows: In Section 2, we describe how the experiments and the analysis have been performed. Section 3 deals with the description of the new proposed algorithm. In Section 4, the results and the major outcomes are given. Finally, in Section 5, conclusions are drawn and defined.

\section{MATERIALS AND METHODS}

\subsection{Beads preparation}

Volume calibration was performed using yellow-green carboxylate-modified microspheres $(2 \mu \mathrm{m}$ diameter; Molecular Probes, Eugene, OR). The product was supplied as an aqueous suspension containing $2 \%$ solids and sodium azide $(2 \mathrm{mM}$ final concentration). Beads were ultraclean polystyrene microspheres coated with a hydrophilic polymer containing multiple carboxylic acids for the covalent attachment of ligands. Each sphere contained fluorescent dye molecules in its whole volume and showed little or no photobleaching, even when excited with the powerful illumination required for fluorescence microscopy.

Following the protocol suggested by Molecular Probes, the stock solution (with about $4.6 \times 10^{9}$ beads $/ \mathrm{ml}$ ) was diluted. Considering the planar experimental spreading of the final fluid sample $\left(1 \mathrm{~cm}^{2}\right.$, about 3500 fields of view), in order to obtain about 20 beads in each field of view, a total amount of $7 \times 10^{3}$ beads/ $\mu \mathrm{l}$ was achieved in the suspended solution. Thereafter, a droplet of ProLong Diamond Antifade Mountant (Molecular Probes) was added onto a glass slide (Menzel-Gläser, Braunschweig, D), while a coverslip (Menzel-Gläser) with $1 \mu \mathrm{l}$ of diluted solution was placed down on it. The sample was kept in the dark, at room temperature, for 24 hours.

\subsection{Blood sample preparation and perfusion chamber}

For each experiment, $20 \mathrm{ml}$ of venous blood from medication-free healthy volunteers were collected into Dphenylalanyl-Lprolyl-L-arginine chloromethyl ketone dihydrochloride (PPACK, $50 \mu \mathrm{M}$ final concentration; Calbiochem, La Jolla, CA). Informed written consent was obtained from the healthy blood donors according to the Declaration of Helsinki and the DMS of the Italian Ministry of Health 2 November 2015 (concerning the quality and safety of blood and blood donors). The Ethics Committee CRO-IRCCS Aviano also approved the study. After obtaining the blood samples, $200 \mu \mathrm{l}$ of acid-insoluble fibrillar type I collagen (Sigma Aldrich, St. Louis, MO) was homogeneously distributed onto a coverslip (Menzel-Gläser) for 60 minutes. The coverslip was thus positioned in a flow chamber, forming the bottom wall of an artificial microchannel with a rectangular section (height $0.125 \mathrm{~mm}$, width $15 \mathrm{~mm}$ ). The fluorescent dye quinacrine dihydrochloride (mepacrine, $10 \mu \mathrm{M}$ final concentration; Sigma Aldrich) was added to whole blood to label platelets. A syringe pump (Harvard Apparatus, Boston, MA) aspirated blood from the chamber outlet for a time interval of three minutes at a flow rate $Q=3.5 \mathrm{ml} / \mathrm{min}$ in order to achieve the shear rate $\gamma$ of

$\gamma=\frac{6 Q}{w h^{2}}=1500 \mathrm{~s}^{-1}$,

where $w$ and $b$ are the width and the height of the channel, respectively.

\subsection{Images acquisition}

The perfusion flow chamber was positioned on the stage of a Confocal Laser Scanning Microscope (CLSM, Eclipse TE300; Nikon, Tokyo, J) based on Nipkow disk technology (Ultra View; Perkin Elmer). A 40X oil-immersion objective (NA 1.30; Nikon) was mounted on a piezoelectric driver controlled by the Andor IQ acquisition software (AndorTM Technology, Belfast, $\mathrm{UK})$. Images were obtained and digitalised in real time with a CCD camera (iXonEM+, AndorTM Technology) with a $512 \times$ 512 pixel sensor. The field of view area was $28547.48 \mu \mathrm{m}^{2}$. Confocal optical images were detected using an excitation laser wavelength of $488 \mathrm{~nm}$. The laser power was set in order to achieve an adequate emission intensity and to avoid the pixel intensity saturation of the CCD sensor.

Images were acquired along the $z$-dimension (Figure 1(a)) with $z$-step $\Delta z=0.2 \mu \mathrm{m}$, starting from the bottom, with dark slices as the baseline $(z=1)$ and ending with dark slices above the objects $(z=N)$. Each sequence of the confocal images acquired along the $z$-dimension is called stack (Figure 1(a)).

\subsection{Volume measurement}

In order to evaluate the systematic errors in the state-of-theart measurements, the confocal digital images were elaborated using ImageJ software (http://imagej.nih.gov/ij), subjectively setting an intensity threshold to select the areas covered by the objects in all the images of a stack acquired along the $z$ dimension. The volume was then measured as the sum of the detected areas in the images, multiplied by $\Delta z$ [13]-[15], [17][19]. Adjusting the intensities histogram, volumes were also 

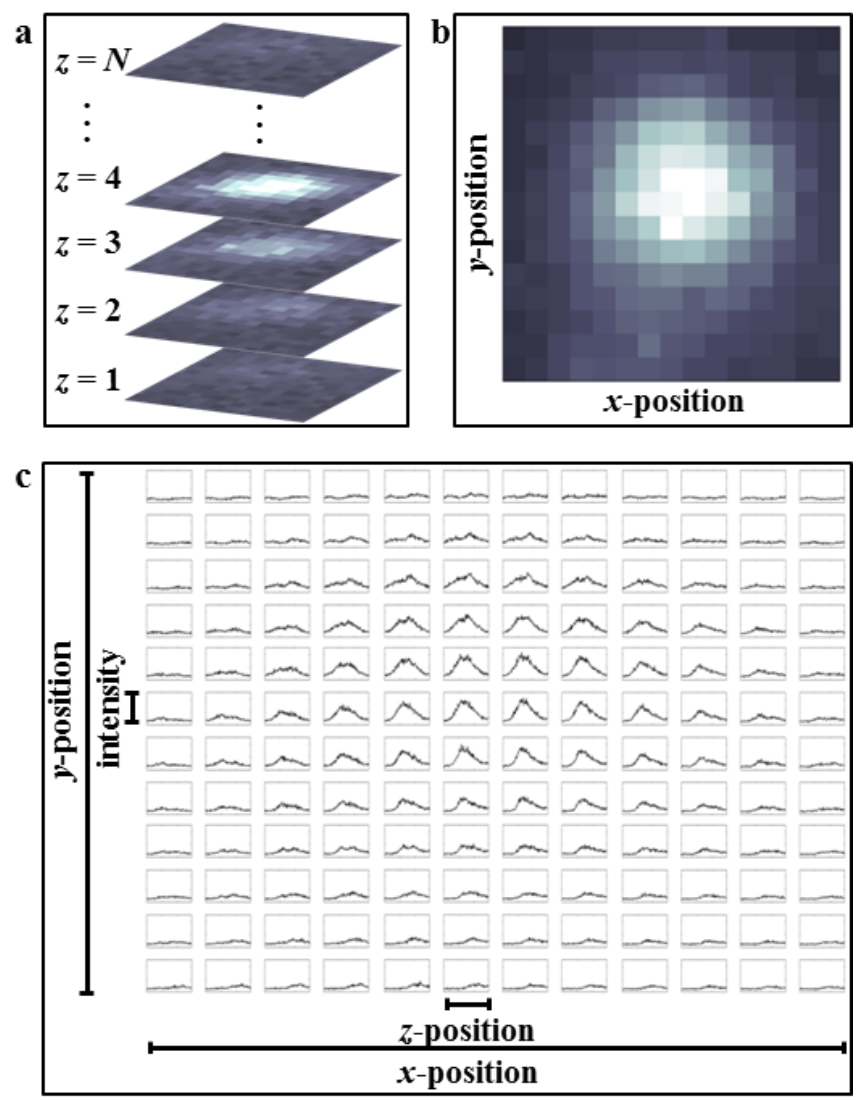

Figure 1. (a) Example of a stack of $N$ confocal images acquired along the $z$ dimension. (b) Bi-dimensional Maximum Intensity Projection (MIP) showing the maximum value that each pixel assumes along the z-dimension. (c) Graphical representation of the three-dimensional matrix defined for a stack: the $x$ - and the $y$-position of each pixel are assigned to the first and second dimensions, respectively, while the $N$ intensity values of each related pixel assumed along the $z$-axis are located in the third dimension.

quantified using Volocity software (Perkin Elmer, Waltham, MA) in a similar way.

The proposed new algorithm was firstly applied to the images of the known volume beads in order to achieve an accurate evaluation of its performance and to find an optimum value for the calibration factor. Consequently, it was applied to small aggregates to verify its robustness with non-spherical samples and finally to platelet large aggregates.

Along the text, all the results are expressed as mean \pm standard deviation, each computed with $n=10$ sample stacks. In order to identify significant differences among the results, the student $t$-test was used.

\section{ALGORITHM DESCRIPTION}

\subsection{Image elaboration and signal extraction}

For each stack (Figure 1(a)), a preliminary control was performed to exclude sequences of images with saturated pixels. For this purpose, the Maximum Intensity Projection (MIP) of all the values (Figure 1(b)) was extracted and visualised.

Thereafter, for each stack, a three-dimensional matrix was defined. The $x$ - and $y$-positions of each pixel were assigned to the first and the second dimensions of the matrix, respectively, while the intensity values of each related pixel assumed along the $z$-axis were allocated in the third dimension of the matrix, starting from the fluorescence value computed in the first slice $(z=1)$ and continuing until the value of the last slice $(z=N)$. A graphical representation of the matrix is shown in Figure 1(c). According to the CCD sensor physical dimensions (a square matrix having $d \times d=512 \times 512$ pixels) and to the number $N$ of slices acquired for a stack, the matrix had the dimensions $d \times d \times N$.

Since the intensities along the $z$-dimension are affected by noise, they have been locally smoothed with a Finite Impulse Response (FIR) low-pass filter to compensate for the high frequency noise. According to the necessity of regularising the $z$-intensities without losing information, the smoothing filter was designed with 13 coefficients and applied to each intensity $z$-signal, performing a weighted mobile averaging. Centring the kernel of coefficients in every z-position, the associated smoothed value $s_{z}$ was obtained from the neighbouring original data $v_{2+i}$ as

$s_{z}=\frac{\sum_{i=-6}^{6} v_{z+i} c_{i}}{\sum_{i=-6}^{6} c_{i}}, z=1,2, \ldots N$,

where $c_{i}$ refers to the coefficients of the filter (Figure 2).

Hence, in order to accomplish the limits that occur in the regularisation of the first and last samples of a generic signal with a kernel of coefficients [34] and to consider the variability of the background fluorescence in all three dimensions, two values of background, $b 1$ and $b 2$ (shown in Figure 2), were quantified as the average of the first six and the last six intensity values of each z-signal.

For every stack, a binary mask was defined, whose dimensions were the $x$ - and $y$-positions of each pixel and were consequently connected to the first and the second dimensions of the related three-dimensional matrix defined before. A value of 1 was assigned to every pixel whose maximum intensity $M$ in the $z$-dimension reached a specific threshold over its background b1 (Figure 3(a)). The threshold, automatically obtained, was considered adaptive because the intrinsic

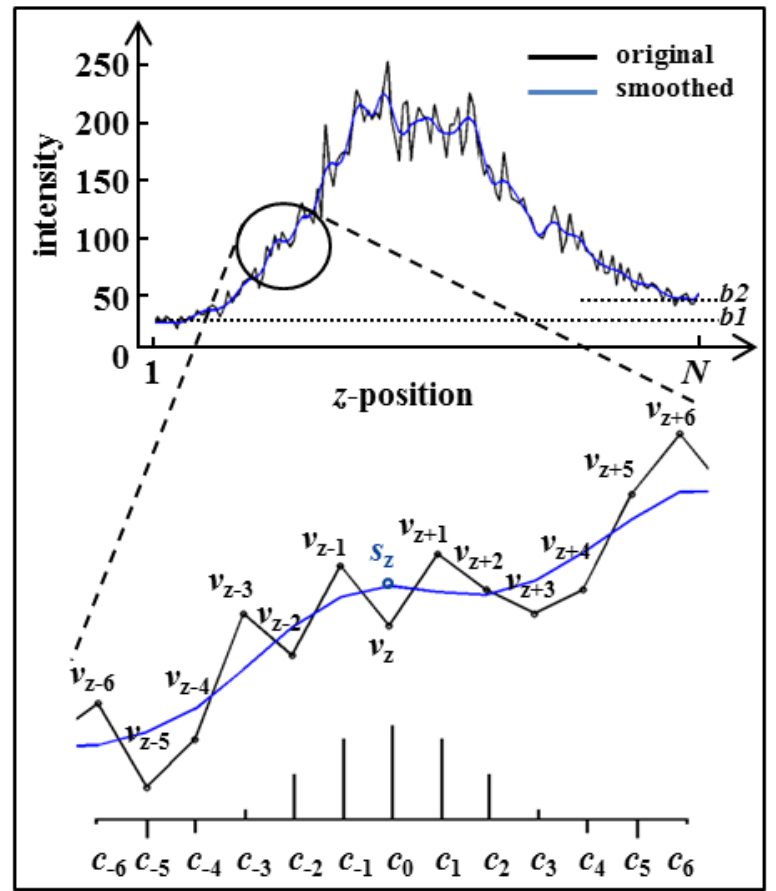

Figure 2. Intensity smoothing by means of the low-pass FIR filter that allows us to obtain the filtered signal $s_{z}$. Two values of background, $b 1$ and $b 2$, are quantified for every $z$-signal as averages of the first and the last six intensity values of the signal, respectively. 

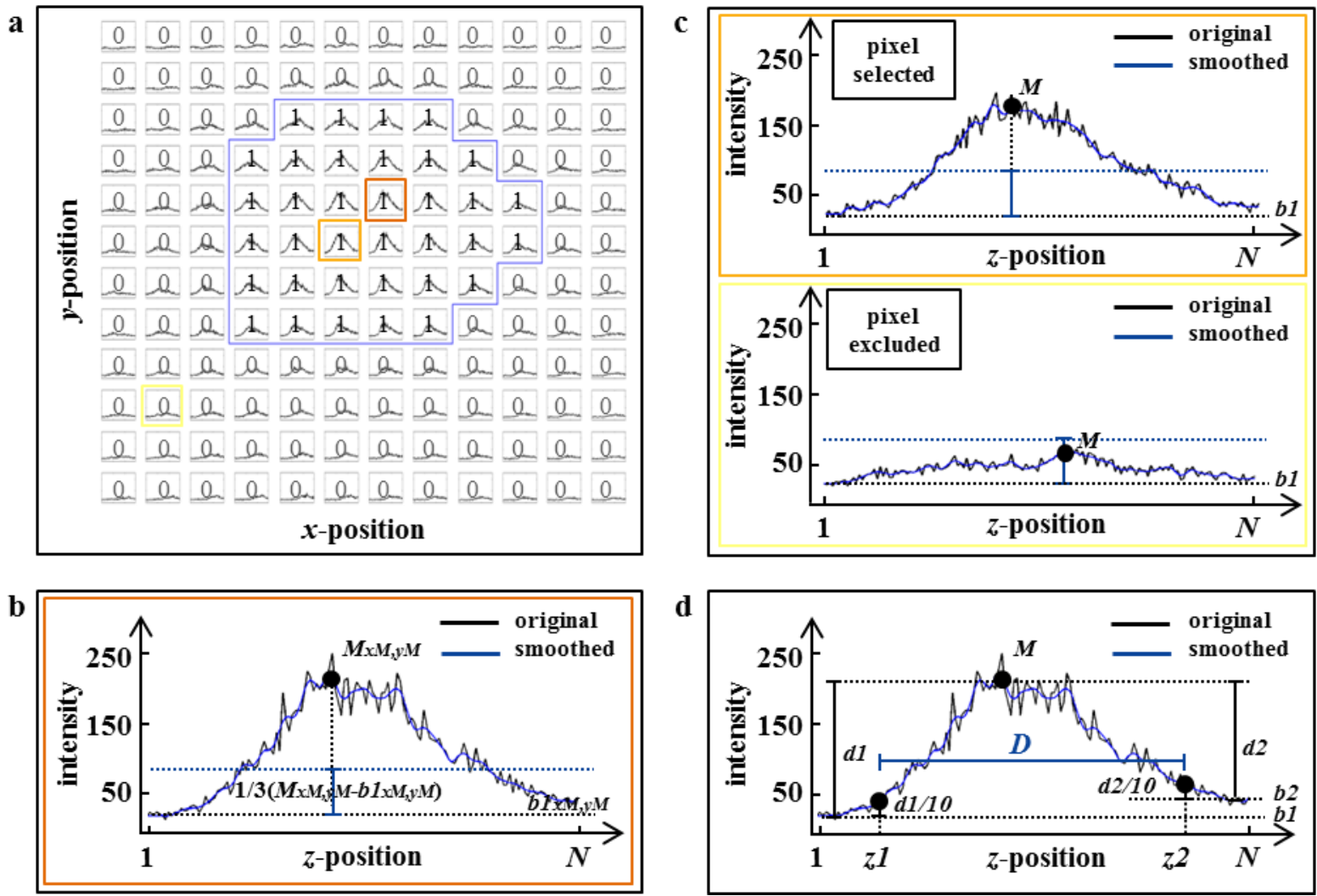

Figure 3. Steps of calibration. (a) Graphical representation of the three-dimensional matrix defined for a sequence of images after applying the binary mask. (b) Calculation of the adaptive threshold. The threshold is set considering $1 / 3$ of the elevation of the maximum peak $M_{x M, y M}$ over its background $b 1_{x M, y M}$. (c) Example of a selected pixel (top) whose $z$-signal shows a peak of intensity $M$ that raises over the threshold. Example of a pixel excluded (bottom) because its related $z$-signal does not go beyond the threshold. (d) Definition of the axial distance $D$ along $z$-dimension, for a generic $z$-signal related to a generic $x$ - and $y$ position. The two $z$-points ( $z 1$ and $z 2)$ are identified as the points whose intensity reaches the $10 \%$ of the related peak $M$, with respect to the backgrounds $b 1$ and $b 2$.

variability of the behaviour of biological samples in light emission does not allow us to set a fixed intensity threshold. Considering the maximum intensity peak $M_{x M y M}$ among all the intensity peaks in the three-dimensional matrix (Figure 3(a) and Figure 3(b)) as a reference value, each pixel value in the binary mask was set to 1 if the related intensity peak $M$ over its background $b 1$ reached at least $1 / 3$ of the reference value $M_{x M, y M}-b 1_{x M, y M}$ (Figure 3(a), Figure 3(b), and Figure 3(c)). Structuring the binary mask as described, it was possible to identify only the $x$ - and the $y$-positions related to a real presence of volume, excluding those affected by the presence of background, noise, and artefacts. The expression used to define the binary mask is

$\operatorname{mask}_{j, k}=\left\{\begin{array}{rr}1 & \text { if } \frac{M_{j, k}-b 1_{j, k}}{M_{x M_{y},}-b 1_{x M y M}}>\frac{1}{3}, j, k=1,2, \ldots d, \\ 0 & \text { elsewhere }\end{array}\right.$

where $j$ and $k$ are the indexes identifying the generic $x$ - and $y$ positions.

\subsection{Calibration of the $z$-elongation}

After having applied the binary mask to the threedimensional matrix, a bi-dimensional matrix was initialised to attribute to every pixel the height of the volume portion located there.

Referring to Figure 3(d), the positions $z 1$ and $z 2$ defined the distance $D=z^{2}-z_{1}$, which had to be calibrated according to their related signals of intensity. For each intensity signal, z.1 and $z^{2}$ were measured starting from the first level of background b1, advancing forward along the signal, and from the second value of background $b 2$, moving backward. Considering the intensity gaps $d 1=M-b 1$ and $d 2=M-b 2$, the two points along the $z$-axis were chosen as the z-positions whose intensity reached an elevation of $d 1 / 10$ and $d 2 / 10$, respectively. The height of the volume portion for each $x$ - and $y$-position was first quantified as the product of the z-distance $D$ with the ratio between the intensity peak and the maximum among all the intensity peaks, related to their background values. Afterwards, the quantity thus obtained was multiplied by an experimental optimal factor $\hat{C}$ estimated as described in Section 4 . The heights $h_{j, k}$ associated with each $x$ - and $y$-position were evaluated as

$b_{j, k}=\hat{C} D_{j, k} \frac{M_{j, k}-b 1_{j, k}}{M_{x M, y M}-b 1_{x M, y M}}, j, k=1,2, \ldots d$,

where $h$ and $D$ are expressed in $\mu \mathrm{m}$ and $\hat{C}$ is the optimal calibration factor, obtained by means of an iterative computation applied on beads stacks in order to satisfy, at the same time, a z-dimensional condition and a volumetric condition, as specified in the following subsection. The described calibration model was applied to small platelets aggregates and finally to more complex aggregates. According to the physical dimension of pixel $r=0.33 \mu \mathrm{m}$, the expression for the final total platelet thrombi volume $V$ of a stack was quantified as

$V=r^{2}\left(\sum_{j=1}^{d} \sum_{k=1}^{d} b_{j, k}\right)$,

where the result is expressed in $\mu \mathrm{m}^{3}$. 

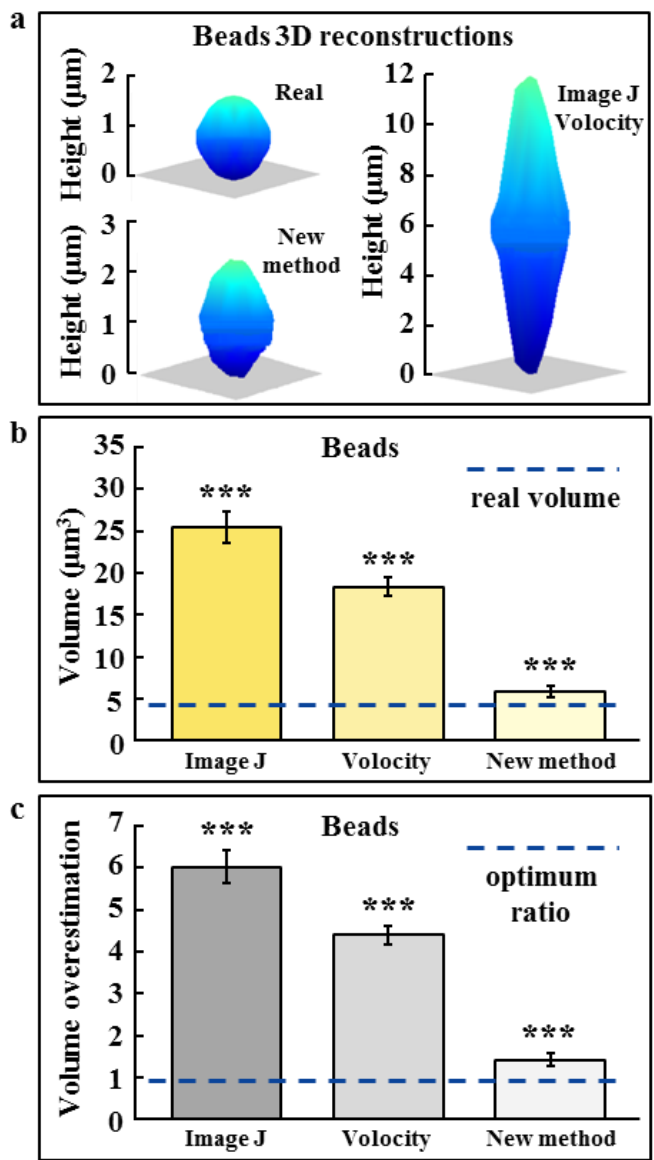

Figure 4. Calibration and measurement results. (a) 3D rendering of a representative bead (top, on the left). 3D reconstruction of the same bead performed with benchmark methods (on the right) and obtained using the new method (bottom, on the left). (b) The beads' absolute volume quantification performed using ImageJ $\left(25 \pm 1 \mu \mathrm{m}^{3}\right)$, Volocity $(18.3 \pm$ $\left.0.8 \mu^{3}\right)$, and the new proposed method $\left(5.9 \pm 0.6 \mu \mathrm{m}^{3}\right)$. Measurement techniques appear significantly different among each other, as confirmed by the $t$-test: $p$ value $<0.001\left({ }^{* * *}\right)$. The dashed line indicates the real volume of each single bead $\left(4.19 \mu^{3}\right.$ ). (c) Bead volume overestimation, with reference to the real volume, obtained using ImageJ $(6.1 \pm 0.3)$, Volocity $(4.3 \pm 0.2)$, and the new proposed method $(1.4 \pm 0.1)$. All the results are expressed as mean \pm standard deviation. The $p$ value remains $<0.001(* * *)$. The dashed line indicates the unitary optimum ratio, which represents perfect matching.

\section{RESULTS}

\subsection{Optimal calibration factor estimation}

The optimal calibration factor $\hat{C}$ was estimated from $n=10$ confocal images sequences representing a single bead of known volume $4.19 \mu \mathrm{m}^{3}$. In particular, $\hat{C}$ was obtained with an iterative computation, which minimised the error function defined as the absolute difference between the real bead volume and the estimated one. The iterations stopped when the maximum height of each bead was at least equivalent to its real diameter ( $z$-dimensional condition) and the correspondent volume was at least equal to its real volume (volumetric condition). The optimum factor resulted in $\hat{C}=0.10 \pm 0.01$.

\subsection{Calibration with fluorescent beads}

In order to validate the model on a different dataset from that which was outlined in the previous subsection, calibration with the optimal factor $\hat{C}$ was applied on a further set of $n=10$ bead stacks that had not been previously used in order to verify the robustness of the method. Figure 4(a) and Figure 4(b) report the numerical estimation and a graphical representation of a single bead volume performed following the procedures presented in Section 2. The results were compared with those obtained using the ImageJ and Volocity software packages. As shown in Figure 4(b), the bead volumes were quantified applying the model calibration settings $5.9 \pm 0.6 \mu \mathrm{m}^{3}$, while the volumes calculated using ImageJ and Volocity were $25 \pm 1 \mu \mathrm{m}^{3}$ and $18.3 \pm 0.8 \mu \mathrm{m}^{3}$, respectively. All three methods appear significantly different, as confirmed by the $t$-test: the $p$ value, for all the comparisons, is less than 0.001. Each single volume was evaluated assuming the real known volume $\left(4.19 \mu \mathrm{m}^{3}\right)$ of a single bead as a reference.

Defining the volume overestimation as the ratio between the measured value and the real one, it has been quantified as $6.1 \pm$ 0.3 with ImageJ, $4.3 \pm 0.2$ with Volocity, and only $1.4 \pm 0.1$ with the new proposed algorithm (Figure 4(c)). A perfect reconstruction would yield to the optimal unitary ratio of overestimation (the dashed line in Figure 4(c)). The proposed method permits the significant reduction of the overestimation error and to obtain realistic volume measurements and representation. The data continues to be significantly different, because each ratio is referred to the same real absolute volume: The $p$ value for all the population comparisons remains less than 0.001 .

\subsection{Platelet aggregates}

The algorithm was then applied to $n=10$ confocal stacks related to small aggregates of platelets (small groups of platelets), i.e. from 3 to 10 , that adhere to the substrate as a mono-layer and whose number can be clearly observed and determined. For each small aggregate, it was thus possible to estimate a realistic absolute volume, multiplying the number of platelets observed by the representative volume of a platelet, considering its semi-spherical discoidal shape and its average diameter of $2 \mu \mathrm{m}$ [35]. In the case of small aggregates, the volume overestimation is $5.5 \pm 0.8$ with ImageJ, $4.3 \pm 0.8$ with Volocity, and $1.3 \pm 0.2$ with the proposed method, as shown in Figure 5(a). This confirms the robustness of the algorithm. The new value thus obtained is significantly different to those estimated using the traditional measurement methodology, with a related $p$ value that is less than 0.001. Figure 6(a) visibly highlights the abovementioned differences measuring and reconstructing the volume of a representative small aggregate of five platelets.

The last step in the proposed method was represented by its application to $n=10$ stacks of images of multi-layer aggregates, composed of a considerable number of platelets. Due to the clear impossibility of accurately pre-estimating a real reference volume, the measurements were expressed as the overestimation achieved using benchmark methods with reference to the measurement performed using the new algorithm. This expression of the results as an overestimation among the methods has also been done for the measurements presented in the previous subsections related to beads and small aggregates (Figure 5(b) and Figure 5(c)).

According to this approach, the accuracy and the repeatability of the proposed calibration algorithm were confirmed by the maintenance of comparable overestimation ratios. The overestimation measured using ImageJ is, in fact, 4.2 \pm 0.2 for beads, $4.1 \pm 0.5$ for small platelet aggregates, and 3.9 \pm 0.6 for large platelet aggregates. This means that the 
metrological compatibility between the measurements of overestimation is respected (Figure 5(b)). Similarly, considering the ratios refer to the Volocity overestimation of each volume, the results obtained are $3.1 \pm 0.2$ for beads, $3.1 \pm 0.6$ for small aggregates, and $2.9 \pm 0.5$ for large aggregates (Figure $5(\mathrm{c})$ ). The overestimation of the benchmark methods is thus proven by compatible intervals. Figure 6(b) compares two representative $3 \mathrm{D}$ reconstructions of large platelet aggregates, obtained with the commercial method and with the proposed calibration algorithm.

\subsection{Uncertainty evaluation}

The uncertainty on the volume estimation has been evaluated by means of the propagation of uncertainty outlined in equation (3), equation (4), and equation (5).

In particular, the main contributions of uncertainty are related to three uncertain quantities: the identification of the intensity threshold for the binary mask, as expressed in equation (3); the identification of intensity thresholds $d_{1}$ and $d_{2}$ to obtain the distance $\mathrm{D}$ in equation (4); and the uncertainty on $\hat{C}$.

Regarding the threshold for the binary mask, the uncertainty in extracting the pixels belonging to the base area depends on the quantisation of the intensities $M_{j, k}$ and $b 1_{j, k}$ in (3); since the intensities are quantised in 255 levels, the quantisation uncertainty assumes a uniform distribution in $[-1,1]$,

$u\left(M_{j, k}\right)=u\left(b 1_{j, k}\right)=1 / \sqrt{3}$.

Thus, the uncertainty in the volume estimation due to the uncertainty in the binary mask can be expressed as

$\left.u(V)\right|_{\text {mask }}=$

$\sqrt{\left(\sum_{j, k} \frac{\partial V}{\partial M_{j, k}} u\left(M_{j, k}\right)\right)^{2}+\left(\sum_{j, k} \frac{\partial V}{\partial b 1_{j, k}} u\left(b 1_{j, k}\right)\right)^{2}}$.

In order to evaluate the two sensitivities in equation (7), we varied the values of $M_{j, k}$ and $b 1_{j, k}$ around the operating points, and we evaluated the change in the readout of the volume estimated. The two sensitivities result in $0.02 \mu \mathrm{m}^{3} / \mathrm{step}$ for beads, $0.05 \mu^{3} /$ step for small aggregates, and $0.95 \mu \mathrm{m}^{3} /$ step for large aggregates.

In the same way, for the uncertainty in the height estimation, we calculated the uncertainty of the volume related to intensities $d_{1}$ and $d_{2}$ in (4). Since $d_{1}$ and $d_{2}$ are, again, intensities quantised in 255 levels, their uncertainty is the same as in equation (6), and the contribution to uncertainty on volume due to height estimation is thus

$\left.u(V)\right|_{\text {height }}=$

$\sqrt{\left(\sum_{j, k} \frac{\partial V}{\partial d 1_{j, k}} u\left(d 1_{j, k}\right)\right)^{2}+\left(\sum_{j, k} \frac{\partial V}{\partial d 2_{j, k}} u\left(d 2_{j, k}\right)\right)^{2}}$.

The sensitivities result in $0.03 \mu \mathrm{m}^{3}$ /step for beads, 0.14 $\mu \mathrm{m}^{3}$ /step for small aggregates, and $1.73 \mu \mathrm{m}^{3} /$ step for large aggregates.

Referring to the uncertainty in the volume estimation due to $\hat{C}$ extraction, we note that this calibration coefficient is a multiplying factor in equation (4), so the uncertainty in volume due to $\hat{C}$ is

$\left.u(V)\right|_{\hat{C}}=\frac{u(\hat{C})}{\hat{C}} V$,

where $u(\hat{C})=0.01$ has been evaluated as the standard deviation of $\hat{C}$, as reported in section 4.1.
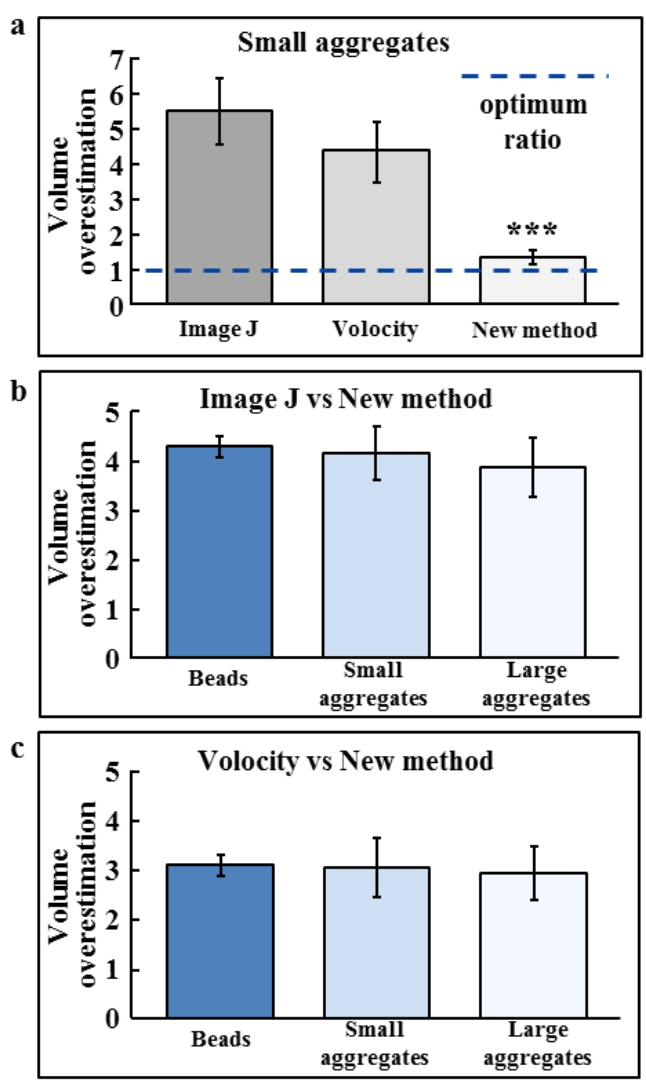

Figure 5. (a) Platelet small aggregates volume overestimation ( $n=10$ aggregates stacks), with reference to their real volume, computed with ImageJ $(5.5 \pm 0.8)$, Volocity $(4.3 \pm 0.8)$, and the new proposed method $(1.3 \pm$ 0.2 ). The last result is significantly different to those estimated using the benchmark methods, with a $p$ value $<0.001(* * *)$. The dashed line indicates the unitary optimum ratio, which represents perfect matching. (b) Volume overestimation with Image with respect to the volume quantified with the new method ( $n=10$ stacks): $4.2 \pm 0.2$ for beads, $4.1 \pm 0.5$ for platelet small aggregates, and $3.9 \pm 0.6$ for large aggregates. (c) Volume overestimation with Volocity with respect to the volume quantified with the new method ( $n$ = 10 stacks) measures $3.1 \pm 0.2$ for beads, $3.1 \pm 0.6$ for small aggregates, and $2.9 \pm 0.5$ for platelet thrombi. The results are expressed as mean \pm standard deviation. All the intervals are metrologically compatible.

The overall uncertainty in the volume finally results in

$u(V)=\sqrt{\left(\left.u(V)\right|_{\text {mask }}\right)^{2}+\left(\left.u(V)\right|_{\text {height }}\right)^{2}+\left(\left.u(V)\right|_{\hat{C}}\right)^{2}}$

The last term in a previous equation prevails since the relative uncertainty on $\hat{C}$ is $10 \%$; thus, the overall uncertainty is in the order of $10 \%$, as shown in Table 1.

Table 1: Estimated volumes for different objects and their uncertainties.

\begin{tabular}{lcc}
\hline & Volume $\left(\mu \mathrm{m}^{3}\right)$ & Uncertainty $\left(\mu \mathrm{m}^{3}\right)$ \\
\hline Beads & 5.9 & 0.6 \\
Small aggregate \#1 & 13.3 & 1.3 \\
Small aggregate \#2 & 13.8 & 1.4 \\
Small aggregate \#3 & 11.7 & 1.1 \\
Small aggregate \#4 & 23 & 2.3 \\
Small aggregate \#5 & 28 & 2.8 \\
Small aggregate \#6 & 16.7 & 1.7 \\
Large aggregate \#1 & 122 & 12 \\
Large aggregate \#2 & 355 & 35 \\
Large aggregate \#3 & 70 & 7 \\
\hline
\end{tabular}



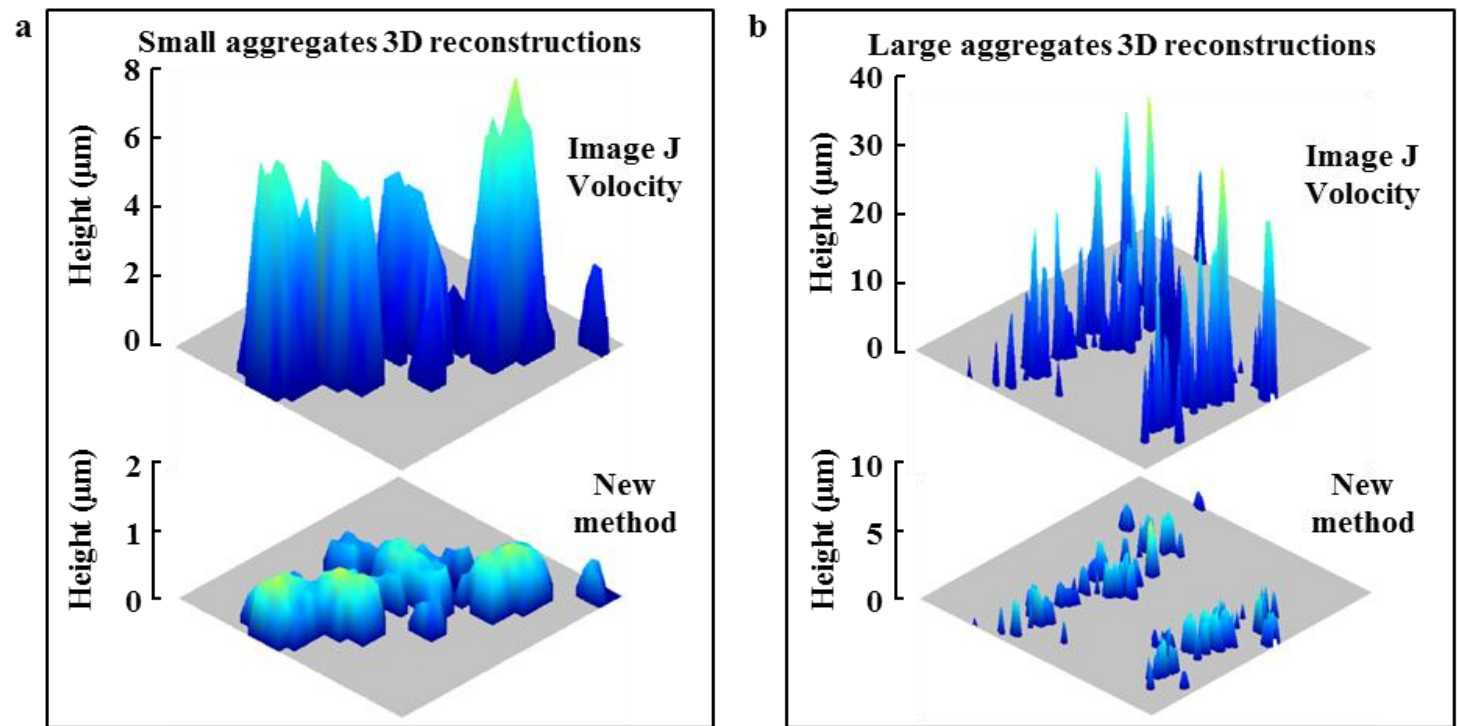

Figure 6. (a) 3D reconstruction of a small aggregate composed of five platelets, obtained using the benchmark methods (top) and the proposed new method (bottom). (b) 3D reconstruction of a cluster of platelet thrombi, achieved with the benchmark methods (top) and with the proposed new method (bottom).

Despite that the uncertainty of the proposed method seems high, it allows us to obtain considerable improvements in terms of measurement reliability and accuracy compared to the traditional methods used to quantify thrombus volume. It is important to specify that, in any case, all the measurements regarding the biomedical field require the critical interpretation and confirmation of the physician due to the high intra- and inter-variability of the patients. Consequently, while the inaccuracy of 400-600 \% with the benchmark methods makes them inapplicable in real medical cases, we can affirm that the uncertainty of $10 \%$ on volume measurements does not represent a problem with regard to the applicability of our method in clinics.

\section{CONCLUSION}

The accuracy and the standardisation of thrombus volume measurements starting from confocal microscope images have always been open and problematic issues. In the specific biological field of blood coagulation and cardiovascular diseases, the possibility of reconstructing and measuring the individual platelet thrombi formed on different adhesive surfaces is of considerable importance for studying platelet functions and the dynamic processes that involve them. The proposed method allows for the fast and accurate compensation of distortion effects and of the related volume overestimation. The algorithm described computes a realistic and accurate volume estimation offering new perspectives in terms of standardisation, inter-laboratory communication, calibration of other devices, medical diagnostics, measurements reliability, and fluid dynamic models definition.

The obtained results aim at compensating for the systematic error arising when a volume is estimated from confocal stacks of images with benchmark commercial methods like ImageJ or Volocity. The analysis indicates that the overestimation of all the benchmark methods is proven by compatible intervals for all the populations of considered objects (beads, small platelet aggregates, and large platelet aggregates). The overestimation error could be considered acceptable and treated as a common offset between measurements only in investigations about the functional effect of a substance. In medical diagnostics, instead, this overestimation is not acceptable (e.g. in tumour mass estimation). Hence, it is mandatory to compute a realistic and accurate volume measurement. Considering the instrumental benchmark that is described herein, the new fast calibration algorithm presented can be positively applied to different biological objects, to those characterised by an irregular shape, or to clusters of objects.

\section{REFERENCES}

[1] R. Eils, C. Athale, Computational imaging in cell biology, J. Cell Biol. 161 (2003) pp. 477-481.

[2] Y. Masutani, H. MacMahon, K. Doi, Computerized detection of pulmonary embolism in spiral CT angiography based on volumetric image analysis, IEEE Trans. Med. Imaging 21 (2002) pp. 1517-1523.

[3] J. Dehmeshki, X. Ye, H. Amin, M. Abaei, X. Lin, S.D. Qanadli, Volumetric quantification of atherosclerotic plaque in CT considering partial volume effect, IEEE Trans. Med. Imaging, 26 (2007) pp. 273-282.

[4] E. K. Kollorz, D. A. Hahn, R. Linke, T. W. Goecke, J. Hornegger, T. Kuwert, Quantification of thyroid volume using 3-D ultrasound imaging, IEEE Trans. Med. Imaging 27 (2008) pp. 457-466.

[5] D. G. Kang, D. C. Suh, J. B. Ra, E. H. K. Stelzer, Threedimensional blood vessel quantification via centerline deformation, IEEE Trans. Med. Imaging 28 (2009) pp. 405-414.

[6] M. M. Frigault, J. Lacoste, J.L. Swift, C.M. Brown, Live-cell microscopy - tips and tools, J. Cell Sci. 122 (2009) pp. 753-767.

[7] J. B. Pawley, Handbook of Biological Confocal Microscopy, Springer, Berlin, 2006, ISBN-13: 978-0387259215.

[8] J. C. Waters, Accuracy and precision in quantitative fluorescence microscopy, J. Cell Biol. 185 (2009) pp. 1135-1148.

[9] S. Hell, G. Reiner, C. Cremer, E. H. K. Stelzer, Aberrations in confocal fluorescence microscopy induced by mismatches in refractive index, J. Microsc. 169 (1993) pp. 391-405.

[10] T. D. Visser, J. L. Oud, Volume measurements in threedimensional microscopy, Scanning 16 (2008) pp. 198-200.

[11] J. M. Murray, P. L. Appleton, J. R. Swedlow, J. C. Waters, Evaluating performance in three-dimensional fluorescence microscopy, J. Microsc. 228 (2007) pp. 390-405.

[12] Y. Hiraoka, J. W. Sedat, D. A. Agard, Determination of threedimensional imaging properties of a light microscope system. 
Partial confocal behavior in epifluorescence microscopy, Biophys. J. 57 (1990) pp. 325-333.

[13] G. L. Mendolicchio, D. Zavalloni, M. Bacci, E. Corrada, M Marconi, C. Lodigiani, P. Presbitero, L. Rota, Z.M. Ruggeri, Variable effect of P2Y12 inhibition on platelet thrombus volume in flowing blood, J. Thromb. Haemost. 9 (2011) pp. 373-382.

[14] N. Pugh, A. M. Simpson, P. A. Smethurst, P. G. de Groot, N. Raynal, R. W. Farndale, Synergism between platelet collagen receptors defined using receptor-specific collagen-mimetic peptide substrata in flowing blood, Blood 115 (2010) pp. 50695079 .

[15] M. Mazzucato, M. R. Cozzi, M. Battiston, M. Jandrot-Perrus, M. Mongiat, P. Marchese, T. J. Kunicki, Z. M. Ruggeri, L. De, Distinct spatio-temporal $\mathrm{Ca} 2+$ signaling elicited by integrin alpha2beta1 and glycoprotein VI under flow, Blood 114 (2009) pp. 2793-2801.

[16] A. Celi, G. Merrill-Skoloff, P. Gross, S. Falati, D. S. Sim, R. Flaumenhaft, B. C. Furie, B. Furie, Thrombus formation: direct real-time observation and digital analysis of thrombus assembly in a living mouse by confocal and widefield intravital microscopy, J. Thromb. Haemost. 1 (2003) pp. 60-68.

[17] Z. M. Ruggeri, J. A. Dent, E. Saldivar, Contribution of distinct adhesive interactions to platelet aggregation in flowing blood, Blood 94 (1999) pp. 172-178.

[18] B. Savage, M. H. Ginsberg, Z. M. Ruggeri, Influence of fibrillar collagen structure on the mechanisms of platelet thrombus formation under flow, Blood 94 (1999) pp. 2704-2715.

[19] B. Savage, F. Almus-Jacobs, Z. M. Ruggeri, Specific synergy of multiple substrate-receptor interactions in platelet thrombus formation under flow, Cell 94 (1998) pp. 657-666.

[20] R. M. Schoeman, M. Lehmann, K. B. Neeves, Flow chamber and microfluidic approaches for measuring thrombus formation in genetic bleeding disorders, Platelets 28 (2017) pp. 463-471.

[21] D. De Zanet, M. Battiston, E. Lombardi, R. Specogna, F. Trevisan, L. De Marco, A. Affanni, M. Mazzucato, Impedance biosensor for real-time monitoring and prediction of thrombotic individual profile in flowing blood, PLoS ONE 12 (2017).

[22] R. M. Schoeman, K. Rana, N. Danes, M. Lehmann, J. A. Di Paola, A. L. Fogelson, K. Leiderman, K. B. Neeves, A microfluidic model of hemostasis sensitive to platelet function and coagulation, Cell. Mol. Bioeng 10 (2017) pp. 3-15.

[23] A. Affanni, G. Chiorboli, R. Specogna, F. Trevisan, Uncertainty model of electro-optical thrombus growth estimation for early risk detection, Measurement 79 (2016) pp. 260-266.

[24] A. Affanni, R. Specogna, F. Trevisan, Ex vivo time evolution of thrombus growth through optical and electrical impedance data fusion, J. Phys. Conf. Ser. 459 (2013).
[25] Y. Zheng, J. Chen, M. Craven, N.W. Choi, S. Totorica, A. DiazSantana, P. Kermani, B. Hempstead, C. Fischbach-Teschl, J. A. Lopez, A. D. Stroock, In vitro microvessels for the study of angiogenesis and thrombosis, PNAS - Proc. Natl. Acad. Sci. USA 109 (2012) pp. 9342-9347.

[26] A. Affanni, R. Specogna, F. Trevisan, A discrete geometric approach to cell membrane and electrode contact impedance modeling, IEEE Trans. Biomed. Eng. 59 (2012) pp. 2619-2627.

[27] A. Affanni, R. Specogna, F. Trevisan, Electrical impedance spectroscopy on flowing blood to predict white thrombus formation in artificial microchannels, Proc. of the IEEE I2MTC - International Instrumentation and Measurement Technology Conference, Graz, Austria, 2012, pp. 1477-1480.

[28] A. Affanni, R. Specogna, F. Trevisan, Measurement bench for Impedance Tomography during hemostasis process in whole blood, Proc. of IEEE MeMeA - International Symposium on Medical Measurements and Applications, Bari, Italy, 2011, pp. 80-83.

[29] A. Affanni, R. Specogna, F. Trevisan, Combined electro-optical imaging for the time evolution of white thrombus growth in artificial capillaries, IEEE Trans. Instrum. and Meas. 62 (2013) pp. 2954-2959.

[30] L. G. Puckett, G. Barrett, D. Kouzoudis, C. Grimes, L. G. Bachas, Monitoring blood coagulation with magnetoelastic sensors, Biosens. Bioelectron. 18 (2003) pp. 675-681.

[31] W. S. Nesbitt, S. Giuliano, S. Kulkarni, S. M. Dopheide, I. S. Harper, S.P. Jackson, Intercellular calcium communication regulates platelet aggregation and thrombus growth, J. Cell Biol. 160 (2003) pp. 1151-1161.

[32] M. Roest, A. Reininger, J. J. Zwaginga, M. R. King, J. W. Heemskerk., Flow chamber-based assays to measure thrombus formation in vitro: requirements for standardization, $\mathrm{J}$. Thromb. Haemost. 9 (2011) pp. 2322-2324.

[33] A. L. Plant, L. E. Locascio, W. E. May, P. D. Gallagher, Improved reproducibility by assuring confidence in measurements in biomedical research, Nat. Methods 11 (2014) pp. 895-898.

[34] C. H. Chen, Signal processing handbook, Marcel Dekker, New York, 1988, ISBN 0-8247-7956-8.

[35] J. N. Thon, L. Mazutis, S. Wu, J. L. Sylman, A. Ehrlicher, K. R. Machlus, Q. Feng, S. Lu, R. Lanza, K. B. Neeves, D. A. Weitz, J. E. Italiano, Platelet bioreactor-on-a-chip, Blood 124 (2014) pp.1857-1867. 